\title{
Perioperative Monitoring
}

Editors

GABRIELLA IOHOM

GIRISH P. JOSHI

\section{ANESTHESIOLOGY CLINICS}

www.anesthesiology.theclinics.com

Consulting Editor

LEE A. FLEISHER

September 2021 • Volume 39 • Number 3 